\title{
A NEW LOOK AT MEANS ON TOPOLOGICAL SPACES
}

\author{
PETER HILTON \\ Department of Mathematics \\ University of Central Florida \\ Orlando, FL 32816-1364 USA \\ and \\ Department of Mathematical Sciences \\ SUNY, Binghamton \\ Binghamton, NY 13902-6000 USA
}

(Received June 5, 1997)

\begin{abstract}
We use methods of algebraic topology to study when a connected topological space admits an $n$-mean map.
\end{abstract}

KEY WORDS AND PHRASES: Means, topological spaces, comeans 1991 AMS SUBJECT CLASSIFICATION CODES: 55PXX

\section{INTRODUCTION}

Carathéodory and Aumann (see [1],[2]) were among the pioneers who first considered the question of what path-connected regions $X$ in $\mathbb{R}^{m}$ or $\mathbb{C}^{m}$ could support an n-mean, that is, a map $\mu: X^{n} \rightarrow X$ satisfying

(i) $\mu \Delta=1 ; X \rightarrow X$, where $\triangle$ is the diagonal map $\triangle: X \rightarrow X^{n}$; and

(ii) $\mu \sigma=\mu: X^{n} \rightarrow X$, where $\sigma \in S_{n}$, the symmetric group on $n$ letters, acting on $X^{n}$ by permuting components One of their main concerns was to find out if the existence of such an $n$-mean, $n \geq 2$, implied that $X$ was simply connected

In 1954, Beno Eckmann [4] attacked the question with the tools of algebraic topology He supposed $X$ to be a polyhedron and only required conditions (i), (ii) above up to homotopy One of his principal conclusions was that if $X$ is compact and admits a (homotopy) $n$-mean for all $n$, then $X$ is contractible

In 1962, Eckmann, together with Tudor Ganea and the author, returned to the study of $n$-means in a more general setting (see [5]). Thus the $n$-mean defined in [4] was a morphism in the category $\mathcal{T}_{h}$ of based connected $\mathrm{CW}$-complexes and based homotopy classes of based maps In this generality one was able to exploit the idea of mean-preserving functors. Thus if $\mathcal{C}, \mathcal{D}$ are categories with products and $F: \mathcal{C} \rightarrow \mathcal{D}$ is a product-preserving functor, then $F \mu$ is an $n$-mean in $\mathcal{D}$ for any $n$-mean $\mu$ in $\mathcal{C}$ Moreover, one could also examine the dual question of the existence of $n$-comeans

It turns out that the concept of $P$-local objects and $P$-localization, where $P$ is a family of primes, and the results related to these concepts in the categones $\mathcal{T}_{h}$ and $\mathcal{N}$, the category of nilpotent groups (see [6]), enable one to simplify many arguments in [5] and to extend the results of that paper

\section{MEANS IN THE CATEGORY OF GROUPS}

Let $\mathcal{G}$ be the category of groups Let $n$ be an integer, $n \geq 2$, and let $P$ be the family of primes $p$ such that $p \nmid n$ We then prove

THEOREM 2.1. The group $G$ admits an $n$-mean $\mu$ in $\mathcal{G} \Leftrightarrow G$ is commutative and $P$-local In that case, if we write $G$ additively, $\mu$ is given by 


$$
\mu\left(g_{1}, g_{2}, \cdots, g_{n}\right)=\frac{1}{n}\left(g_{1}+g_{2}+\cdots+g_{n}\right) .
$$

PROOF. Note first that if $G$ is commutative, then $G$ is $P$-local if and only if $G$ admits unique division by $n$ It is then plain that (21) defines an $n$-mean on $G$

Conversely, let $\mu$ be an $n$-mean on $G$ For $g, h \in G$ (at this stage, we write $G$ multiplicatively), set $\mu(g, e, \cdots, e)=\gamma, \mu(h, e, \cdots, e)=\delta \quad$ Then, by condition (ii),

$$
\mu(e, g, \cdots, e)=\cdots=\mu(e, e, \cdots, g)=\gamma,
$$

so that, by condition (i),

$$
g=\mu(g, g, \cdots, g)=\gamma^{n} .
$$

Similarly, $h=\delta^{n} \quad$ But $\mu(g, h, \cdots, e)=\gamma \delta, \mu(h, g, \cdots, e)=\delta \gamma$, and $\mu(g, h, \cdots, e)=\mu(h, g, \cdots, e)$ Thus $\gamma$ commutes with $\delta$, so that $g$ commutes with $h$ and $G$ is commutative To show that $G$ is $P$-local it remains to show that $n^{\text {th }}$ roots are unique in $G$. But, again using properties (i) and (ii), we conclude that $\mu\left(g^{n}, e, \cdots, e\right)=\mu(g, g, \cdots, g)=g$, so that $g$ is determined by $g^{n} \quad$ Thus $G$ is commutative and $P$ local and, writing additively, we have

$$
\mu\left(g_{1}, g_{2}, \cdots, g_{n}\right)=\sum_{i=1}^{n}\left(g_{i}, 0, \cdots, 0\right)=\sum_{i=1}^{n} \frac{1}{n} g_{2}=\frac{1}{n}\left(g_{1}+g_{2}+\cdots+g_{n}\right) .
$$

COROLLARY 2.2. Let $G$ be a group and let $n_{1} \geq 2, n_{2} \geq 2$ be integers Then $G$ admits an $n_{1} n_{2}$-mean if and only if $G$ admits an $n_{1}$-mean and an $n_{2}$-mean

\section{MEANS IN THE CATEGORY $\boldsymbol{T}_{h}$}

Let $X$ be a connected CW-complex with base point. We prove, with $n, P$ as in Section 2,

THEOREM 3.1. Suppose $X$ admits an $n$-mean $\mu: X^{n} \rightarrow X$ in $\mathcal{T}_{h}$ Then $X$ is a $P$-local commutative $H$-space

PROOF. We regard the $i^{\text {th }}$ homotopy group $\pi_{\imath}$ as defining a product-preserving functor from $\mathcal{T}_{h}$ to $\mathcal{G}$ Then $\mu_{*}=\pi_{2} \mu:\left(\pi_{2} X\right)^{n} \rightarrow \pi_{2} X$ is an $n$-mean in $\mathcal{G}$ It follows that $\pi_{2} X$ is commutative (this is only significant for $2=1)$ and $P$-local and that $\mu$, has the form (2 l).

Let $i_{1}: X \rightarrow X^{n}$ be the obvious embedding. Then $\left(\mu i_{1}\right)_{*}$ is the endomorphism $g \mapsto \frac{1}{n} g$ of the commutative $P$-local group $\pi_{2} X$ It follows that $\left(\mu i_{1}\right)_{z}$ is an automorphism for all $i$, so that $\mu \imath_{1}$ is a selfhomotopy-equivalence of $X$ Let $\rho: X \rightarrow X$ be homotopy inverse to $\mu i_{1}$. Let $\imath_{12}^{\prime}: X^{2} \rightarrow X^{n}$ be the obvious embedding and let $m=\rho \mu_{12}: X^{2} \rightarrow X$. Then it is easy to see that $m$ is a commutative $H$-structure on $X$ We conclude that $X$ is a $P$-local commutative $H$-space

From Theorem 2.1 we deduce, more easily than in [5],

THEOREM 3.2. If a compact, connected polyhedron $X$ admits an $n$-mean for some $n \geq 2$, then $X$ is contractible.

PROOF. Since the homotopy groups of $X$ are $P$-local, so are the homology groups $H_{2} X, i \geq 1$ (see [6]). Now Browder has shown [3] that a compact, connected polyhedron $X$ which is an $H$-space satisfies Poincare duality. Thus, if $X$ is not contractible, there exists a positive dimension $N$ which contains the universal class giving rise to the duality isomorphism $H_{z}(X) \simeq H^{N-\imath}(X)$. In particular, $H_{N} X=\mathbb{Z}$, but this is absurd, since $\mathbb{Z}$ is not divisible by $n$

REMARK 1. We have not invoked commutativity of the $H$-structure in this argument If we do so, we may apply a theorem of Hubbuck showing that $X$ would be equivalent to a product of circles, which is also impossible for a non-contractible $P$-local space 
REMARK 2. Theorem 3.2 is delicate The $n$-solenoid is compact and admits an $n$-mean but is not a polyhedron The Eilenberg-MacLane space $K(\mathbb{Q}, m)$ is a polyhedron and admits an $n$-mean for every $n$, but is not compact

We have not proved-and doubt the truth of - the converse of Theorem 31 However, one may readily prove

THEOREM 3.3. If $X$ is a $P$-local, connected, commutative, associative $H$-space, then $X$ admits a unique homomorphic $n$-mean. Further, if the connected $H$-space $(X, m)$ admits a homomorphic $n$ mean, then $(X, m)$ is commutative and associative.

The case $n=2$ admits a very neat and precise statement. If $\mu: X^{2} \rightarrow X$ is a 2-mean on $X$, we define $\rho$ as in the proof of Theorem 3.1 as homotopy inverse to $\mu i_{1}$, and $m=\rho \mu$ is a commutative $H$ structure on the $P$-local space $X$, where $P$ is the family of odd primes Conversely, if $m: X^{2} \rightarrow X$ is a commutative $H$-structure on the $P$-local space $X$, we define $\tau$ to be homotopy inverse to $m \triangle: X \rightarrow X$ (notice that $m \triangle$ induces doubling on the homotopy groups of $X$ and is therefore a self-homotopyequivalence). Then $\mu=\tau m$ is a 2 -mean on $X$.

THEOREM 3.4. The function $\mu \mapsto \rho \mu$ sets up a one-one correspondence between 2-means on the $P$-local connected CW-complex $X$ and commutative $H$-structures on $X$

PROOF. If $m=\rho \mu$, then $\mu \Delta=\rho \mu \triangle=\rho$, so $\tau$, defined above, is homotopy inverse to $\rho$ and $\tau m=\mu \quad$ If $\tau \mu=\mu$, then $\tau=u i_{1}$ so, again, $\rho$ is homotopy inverse to $\tau$ and $\rho \mu=m$ Thus the function $m \mapsto \tau m$ is inverse to the function $\mu \mapsto \rho \mu$

\section{THE DUAL STORY}

Whereas the product in a familiar category (like $\mathcal{T}_{h}, \mathcal{G}$ ) takes a familar form essentually independent of the category, the form of the coproduct depends very much on the category in question The three categones which will come into question here are $\mathcal{T}_{h}, \mathcal{G}$, and $\mathcal{A} b$, the category of abelian groups

Let $\mathcal{C}$ be a category admitting finite coproducts, we will write $C \vee D$ for the coproduct of $C$ and $D$ in $\mathcal{C}$ and $C_{n}$ for the coproduct of $n$ copies of $C$ in $\mathcal{C}$. Obvously, the symmetric group $S_{n}$ acts on $C_{n}$, we will write $\nabla: C_{n} \rightarrow C$ for the codiagonal, which is the morphism that concides with the identity on each copy of $C$ in $C_{n}$ Then an n-comean on $C$ is a morphism $\mu: C \rightarrow C_{n}$ such that (i) $\nabla \mu=1: C \rightarrow C$, and (ii) $\sigma \mu=\mu$, for all $\sigma \in S_{n}$ We prove

THEOREM 4.1. In $\mathcal{G}$ only the trival group admits an $n$-comean, $n \geq 2$

PROOF. Let $G$ be a non-trivial group and let $g \in G, g \neq e$ If $\mu: G \rightarrow G_{n}$ is an $n$-comean, $n \geq 2$, then It follows from (i) that $\mu g \neq e$ Now $G_{n}$ is the free product of $n$ copies of $G$, so a non-trival element of $G_{n}$ is uniquely expressible as $h_{\imath_{1}} h_{\imath_{2}} \cdots h_{z_{k}}$, where $G_{(\imath)}$ is the $i^{\text {th }}$ copy of $G$ in $G_{n}, h_{\imath} \in G_{(\imath)}, h_{\imath} \neq e$, and $i_{q} \neq i_{q+1}$, $q=1,2, \cdots, k-1$ Such an element is obviously moved under any permutation $\sigma$ which moves $i_{1}$, so that condition (ii) is violated.

THEOREM 4.2. In $\mathcal{A} b$, the abelian group $A$ admits an $n$-comean, $n \geq 2$, if and only if it admits an $n$-mean In that case $\mu: A \rightarrow A_{n}$ is given by

$$
\mu(a)=\left(\frac{a}{n}, \frac{a}{n}, \cdots, \frac{a}{n}\right) .
$$

PROOF. We note first that, in $\mathcal{A} b, C \vee D=C \oplus D$, so that $A_{n}=A^{n}$ If $A$ admits an $n$-mean, then, by Theorem 2.1, it is clear that (4.1) is an $n$-comean. Suppose conversely that $\mu: A \rightarrow A_{n}$ is an $n$ comean It is then plain from (ii) that $\mu(a)=(\alpha, \alpha, \cdots, \alpha)$ for some $\alpha \in A$ such that, by (i), $n \alpha=a$. It remains to show that division by $n$ is unique in $A$. But

$$
\mu(n a)=(n \alpha, n \alpha, \cdots, n \alpha)=(a, a, \cdots, a),
$$

so that $a$ is determined by na 
REMARK. Note that the situations for means and comeans are very different Means in $\mathcal{G}$ concide with means in $\mathcal{A} b$, on the other hand, there are no non-trival comeans in $\mathcal{G}$ but there are non-trival comeans in $\mathcal{A} b$, and, moreover, the objects in $\mathcal{A} b$ admitting $n$-comeans coincide with those admitting $n$-means

We now study $n$-comeans in $\mathcal{T}_{h}$. Using the same notation as in Theorem 3.1, we prove

THEOREM 4.3. Suppose $X$ is a connected CW-complex admitting an $n$-comean $\mu: X \rightarrow X_{n}$ in $\mathcal{T}_{h}, n \geq 2$ Then $X$ is a simply connected $P$-local commutative $H^{\prime}$-space

PROOF. Now $X_{n}$ is just a bouquet of $n$ copies of $X$ Since $\pi_{1}: \mathcal{T}_{h} \rightarrow \mathcal{G}$ is coproduct-preserving, $\pi_{1} \mu$ is an $n$-comean on the fundamental group $\pi_{1} X$, so that, by Theorem $41, X$ is simply connected Now the homology groups $H_{\imath}, \imath \geq 1$, are coproduct-preservng functors $\mathcal{T}_{h} \rightarrow \mathcal{A} b$, so that, by Theorems 21 and 42 , the homology groups $H_{\imath} X$ are the $P$-local. Since $X$ is simply connected, this implies that $X$ is $P$-local Finally we adopt a line of reasoning entrrely analogous to that in the proof of Theorem 31 to conclude that $X$ admits a commutative $H^{\prime}$-structure $m: X \rightarrow X_{2}$ (Notice that, since $X$ is simply connected, a map $f: X \rightarrow X$ inducing homology isomorphisms is a homotopy equivalence.)

Notice that there are straightforward and valid duals of Theorems 3.3 and 34 On the other hand, Theorem 3.2 does not dualize. For example, the Moore space $M(\mathbb{Z} / 2, m), m \geq 2$, characterized as the unique simply connected homotopy type with $H_{2}=\mathbb{Z} / 2, H_{2}=0, i \geq 3$, is a compact $(m+1)$ dimensional polyhedron which admits an $n$-comean for every odd $n$

\section{REFERENCES}

[1] AUMANN, G, Math. Annalen 111 (1935), 713-730

[2] AUMANN, G, Math. Annalen 119 (1943), 210-215

[3] BROWDER, W, On torsion in $H$-spaces, Ann. Math. (2) 74 (1961), 24-51

[4] ECKMANN, B., Räume mit Mittelbildung, Comm. Math. Helv. 28 (1954), 329-340

[5] ECKMANN, B., GANEA, T and HIITTON, T., Generalized means, Studies in Mathematical Analysis and Related Topics, Stanford University Press (1962), 82-92.

[6] HILTON, P., MISLIN, G. and ROITBERG, J., Localization of nilpotent groups and spaces, Mathematical Studies 15, North Holland (1975) 


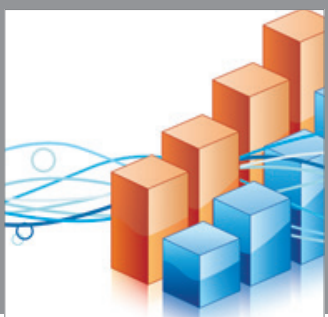

Advances in

Operations Research

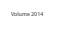

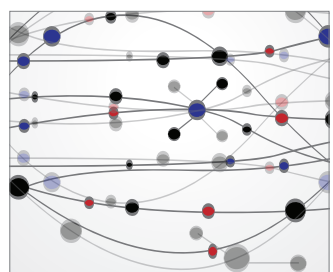

\section{The Scientific} World Journal
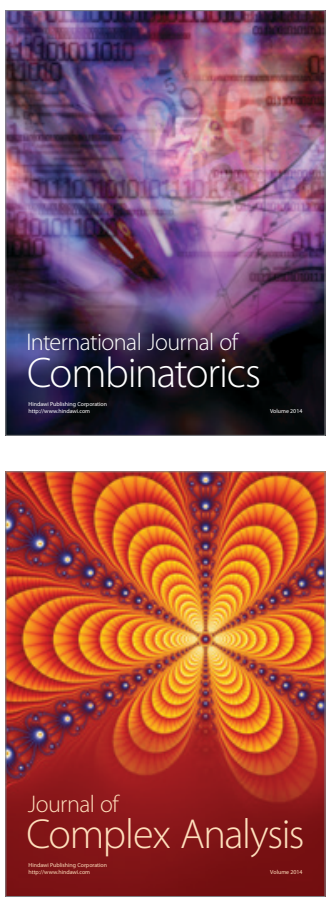

International Journal of

Mathematics and

Mathematical

Sciences
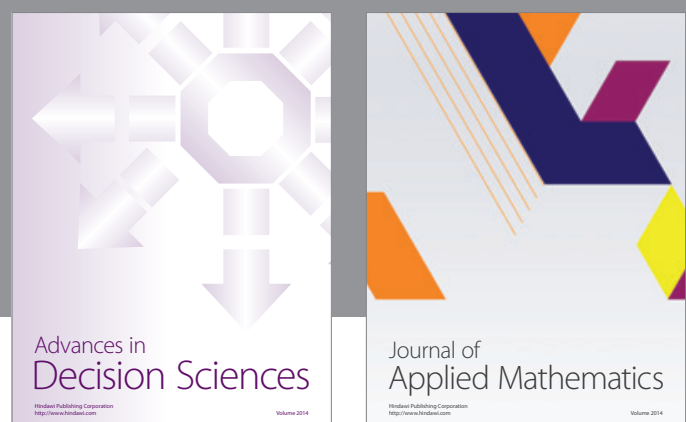

Journal of

Applied Mathematics
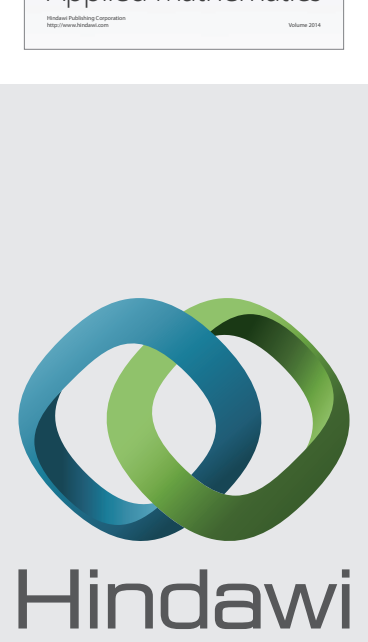

Submit your manuscripts at http://www.hindawi.com
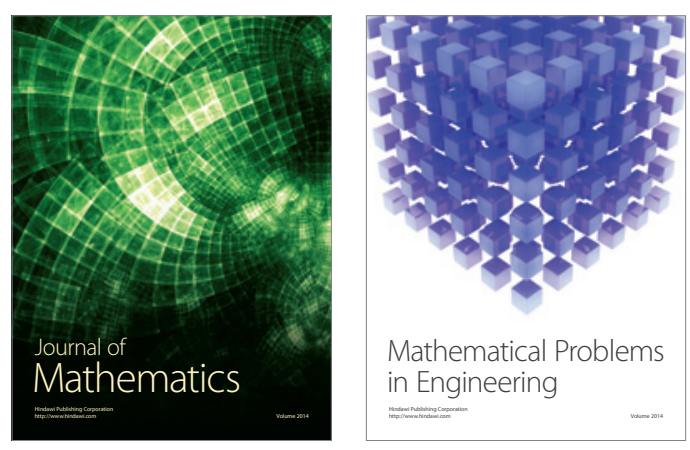

Mathematical Problems in Engineering
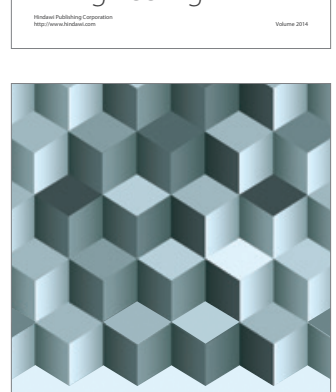

Journal of

Function Spaces
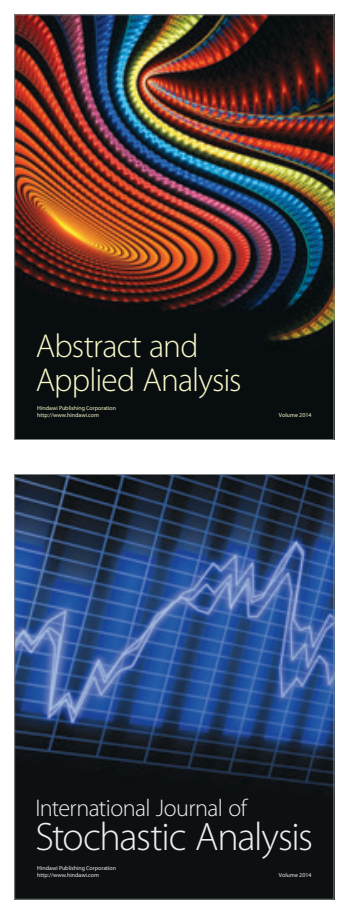

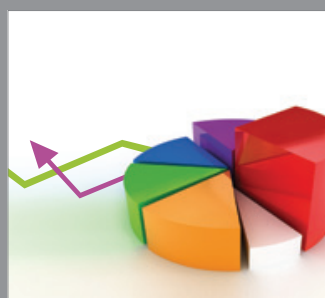

ournal of

Probability and Statistics

Promensencen
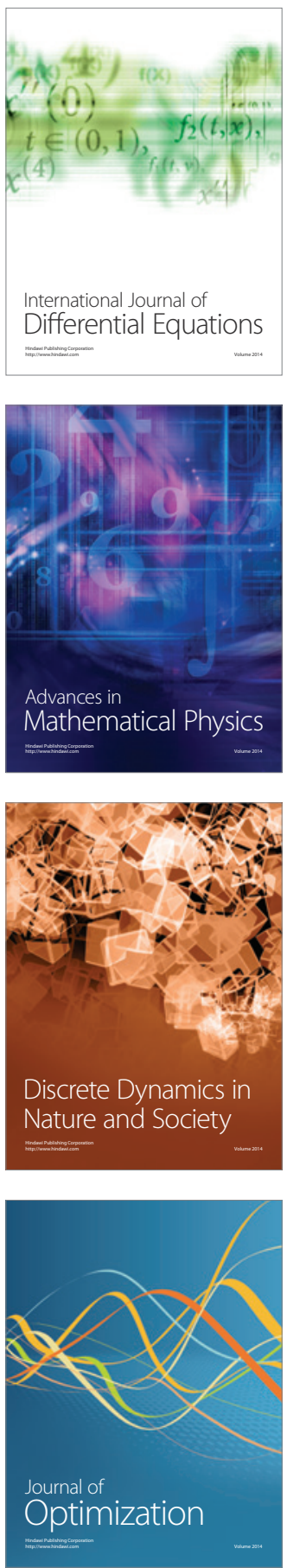